\title{
Knowledge Sharing in Tension: Interacting and Documenting on Stack Overflow
}

\author{
Thomas Hillman, Alena Seredko, Markus Nivala, Tanya Osborne \\ University of Gothenburg, Gothenburg, Sweden \\ \{thomas.hillman, alena.seredko, markus.nivala, tanya.osborne\}@gu.se
}

\begin{abstract}
This exploratory paper examines a tension between interacting and documenting as knowledge sharing tasks on Stack Overflow, a platform that supports informal learning at scale in the domain of programming. The study works with platform data in the form of the text of posts and accompanying metadata along with 16 interviews with users. Drawing on trace ethnography as an approach to maintaining an interpretive stance while combining several types of data, this preliminary analysis discusses two interrelated particularities of the tension. The discussion of these particularities, platform mechanics and competing temporalities, helps to unpack a tension that is both a phenomenon of analytic interest and a member's concern for users of the platform.
\end{abstract}

\section{Author Keywords}

Community question answering; knowledge sharing; informal learning

\section{CSS Concepts}

-Applied computing $\rightarrow$ Education $\rightarrow$ Collaborative learning

-Human-centered computing $\rightarrow$ Collaborative and social computing $\rightarrow$ Empirical studies in collaborative and social computing

-Social and professional topics $\rightarrow$ Professional topics $\rightarrow$ Computing education $\rightarrow$ Informal education

\section{INTRODUCTION}

At the 2020 meeting of Learning @ Scale, it was argued that literature in the area of the conference is dominated by the topics of MOOCs and higher education and that informal online learning communities are underexamined [3]. Responding to the call for research into the particularities of different forms of informal online learning at scale, this study is a preliminary investigation into a tension found on some platforms that support such learning. This tension is between the knowledge sharing tasks of

Preprint version of Hillman, T., Seredko, A., Nivala, M., \& Osborne, T. (2021). Knowledge sharing in tension: Interacting and documenting on Stack Overflow. Proceedings of the Eighth ACM Conference on Learning@ Scale. https://doi.org/10.1145/3430895.3460981 interacting and documenting and it is examined here through the case of the programming knowledge sharing platform Stack Overflow.

\section{CQA and Stack Overflow}

Stack Overflow is an online platform that receives more than 50 million visitors a month seeking and exchanging knowledge about computer programming. In contrast to other forms of knowledge sharing platforms like peerproduced encyclopedia Wikipedia or the discussion forums of Reddit, Stack Overflow follows the Community Question Answering (CQA) structure. This structure means that interaction on the platform is primarily organized in the form of questioning and answering where professional and enthusiast programmers provide solutions to each other's authentic programming problems [4]. The answers provided may only provide a resource for the question asker, as is the case for oft-repeated simple questions [7], but in other cases, they may also be useful for other interested users who can search using both free-text and tags.

Several CQA platforms have emerged over the years including ProfNet, WikiAnswers, Yahoo! Answers, and Answer Garden. Stack Overflow and its sister Stack Exchange sites on other topics share many of the structural features common to the CQA genre. These include user moderation of content through voting and a scoring system that rewards participation [5]. However, unlike some other CQA platforms, Stack Overflow is also structured to allow users to edit each other's posts with greater editing privileges afforded with higher scores known as 'reputation'. Many users can not only comment on posts made by others, but they can also suggest changes by directly editing and, at the highest reputation levels, unilaterally make changes.

\section{Interacting versus documenting on Stack Overflow}

While the question-answer threads structure the knowledge sharing on Stack Overflow, editing and moderation features change the nature of this sharing by potentially turning the threads from short interactions into evolving resources that can be seen as a form of peer-production like pages on Wikipedia. In this sense, there is an orientation towards building a resource with threads that are deemed useful often maintained by the community, while questions or answers deemed to be of low quality are often removed by community members or by scripts run by the platform itself [10]. However, despite this orientation, Stack Overflow remains a site where users can post any question about programming that they like with the expectation of 
receiving help for their problem. This, we argue leads to a tension between the tasks of interacting and documenting that may be implicated on knowledge sharing platforms with a variety structures, but that is particularly visible in the case of CQA platforms like Stack Overflow.

\section{A phenomenon of interest and a member's concern}

As part of the platform, Stack Overflow has a sister site where users can post questions, answers and comments about the main site called meta.stackoverflow. Examining the meta site, it becomes apparent that a tension between the tasks of interacting and documenting is not only a phenomenon of analytic interest, but also a member's concern in the sense suggested by Garfinkel [1]. Numerous threads witness the tension with users claiming various positions with some arguing that Stack Overflow is or should be a curated corpus of high-quality solutions to programming problems and that this project is disrupted by any efforts to answer duplicate, insignificant or otherwise low-quality questions. Users making such arguments can be seen to be prioritizing concerns associated with documentation aspects of knowledge sharing. However, other users routinely argue that it is counter intuitive to invite people to ask questions if the communal project of the site is not to provide them with answers. They can be understood to at least partly prioritize interactional aspects of knowledge sharing and often raise the issue that question askers asking about authentic programming problems that are of low quality according to community norms are often punished through downvotes and post removals.

Given the tension between interacting and documenting is a matter of concern from both an analytic point of view and for users of the platform, this preliminary exploratory study is guided by the question, what specificities of a tension between interacting and documenting on Stack Overflow can be identified?

\section{METHOD}

To examine the tension between interacting and documenting on Stack Overflow several datasets were assembled that include various aspects of the content of interactions on the platform along with metadata such as timestamps and upvotes since the inception of the platform in 2008. These was collected using a combination of the Stack Exchange Structured Query Language (SQL) and Application Programming (API) interfaces. Analysis of the assembled datasets drew on an approach that has been characterized as trace ethnography [2, 6] where an interpretive stance is maintained while numeric and categorical trace data is visualized and analyzed alongside more traditional ethnographic data. In this case, that data consists of the platform interactions of users on the main and meta sites of Stack Overflow along with 16 interviews of between 45 and 60 minutes. These interviews were conducted with members whose experience on the platform can be considered in terms of their scores as ranging from those with amongst the highest and lowest scores available.

\section{PRELIMINARY FINDINGS}

We have already argued that a tension between interacting and documenting is an analytic concern and, as is evident from the discussions on meta.stackoverflow, a member's concern. Examining trace data from the platform along with interviews with users, we seek to begin unpacking that tension and offer a preliminary exploration of its specificities.

\section{Specifying the tension}

Examination of the 16 interviews with users shows a concern for a tension between interacting and documenting in similar ways to those found on meta.stackoverflow. Even amongst users with similarly long histories and scores on the platform, there is an acknowledgement of the tension and disagreement about where the focus of activity should lie. For example, one highly ranked user stated that there is,

"... a common misunderstanding that the purpose of Stack Overflow is for them to get their questions answered and their problems solved, and the idea of Stack Overflow isn't exactly that. It's rather to build a library of questions and answers that will be useful for other people coming back via Google later and this changes how you have to write questions." (4)

However, another well-ranked user describing the kind of communication that takes place on the platform noted that,

"You have a question, I have an answer, it's not a discussion. It's closed ended questions only, not open ended. You are not here to state your opinion, but much more to provide a solution and make sure that the original poster can go on with his or her day." (3)

These extracts exemplify the tension between the activity of sharing knowledge by interacting through questions and answers and by contributing to the peer-production of a library of documentation. However, the interviews also suggest specificities involved in the tension. For the purposes of this preliminary exploratory study, we will focus on platform mechanics and competing temporalities.

\section{Platform mechanics}

Stack Overflow is a platform with a complex set of mechanics. These mechanics are most visible to users in the accumulation of a single reputation score that is described in official Stack Overflow texts as,

"...a rough measurement of how much the community trusts you; it is earned by convincing your peers that you know what you're talking about" [9].

Reputation is generated through a series of mechanisms including upvotes on posts, acceptance of answers to another user's questions, and acceptance of suggested edits to another user's posts. Examining the breakdown of how 
reputation scores were earned for the 16 users interviewed as part of our study, we can see that on average upvotes on posts and acceptance of answers account for $90 \%$ of the score. By contrast, the next most contributing mechanism, when a suggestion on an edit to another's post is accepted, accounts for only $1 \%$ of the score (see Table 1 ).

\begin{tabular}{|l|l|}
\hline Mechanism & Contribution \\
\hline post_upvoted & $72 \%$ \\
\hline answer_accepted & $18 \%$ \\
\hline suggested_edit_approval_received & $1 \%$ \\
\hline
\end{tabular}

Table 1. Top three reputation score contributing mechanisms.

While the proportion of contribution to reputation score of different platform mechanisms depends on several factors including community norms and individual behavior, it is worth noting that the maximum possible score for edits to other users' posts is capped at 1000 and by the removal of the need to have edits approved above certain reputation levels. That is to say that users with reputation scores above the relatively low level of 2000 do not receive any score for edits to most types of posts. In addition, users who post answers or users elected as moderators have the option to turn a thread into a "Community Wiki" where the thread is opened for editing by users with lower reputation scores. Once this change in status is made no reputation score can be accrued from the thread any longer, but it should be noted that at the time of writing, the option had only been only exercised on around $0.5 \%$ of threads on the platform.

The mechanics of reputation score reward tasks that can be upvoted such as posting new questions and answers over the editing of existing posts. In this sense, the mechanics of the platform can be seen to reward certain kinds of interaction over tasks that can be seen to be more strongly associated with maintaining the documentation on the platform. However, while the mechanics of the platform might reward certain types of interaction that increase the amount of posting, users also work to maintain threads as documents where knowledge is shared, sometimes going to great lengths. When discussing an answer, one of the user's interviewed as part of this study remarked that,

"a couple of private messages reached me where there were folks that actually pinged me, either on Twitter or some other platform, that my answer is not complete because things have changed. And they gave me the update answer, or the external information and I had to edit my answer on Stack Overflow." (10)

The user explains that other users had made the effort to find them on other online platforms and request edits be made to old answers to update them to current technical standards.

\section{Competing temporalities}

One particularity of the tension between interaction and documentation on Stack Overflow is mechanisms that reward certain types of interaction over the maintenance of documentation while users also orient to that maintenance and often frame it as the primary knowledge sharing task on the platform. A related particularity is the competing temporalities of these two knowledge sharing tasks.

While maintaining an up-to-date repository of documentation on relevant programming problems can be seen as a long-term project based on a process of refining the available information, answering a question can be seen as a more immediate short-term project. This distinction is exacerbated by the mechanics of the platform that tend to reward a quick pace of interaction. As one interviewee noted in response to a question about their strategies for increasing their reputation score, speed matters:

"there is a lot of CSS-questions asked by new developers that ... they are easy to answer. And if you answer it fast, because there's a lot of other developers on Stack Overflow which just see the latest question and spend time on it, that will answer before you, so you really have to find the answer quite fast." (13)

This answer depicts how the interviewee looks for easy questions that can be answered quickly, a strategy mentioned by several others and discussed on meta.stackoverflow. Answering a question first, is thus seen as increasing the odds of collecting score for upvotes and answer acceptance, however, it also opens the possibility for passive reputation growth.

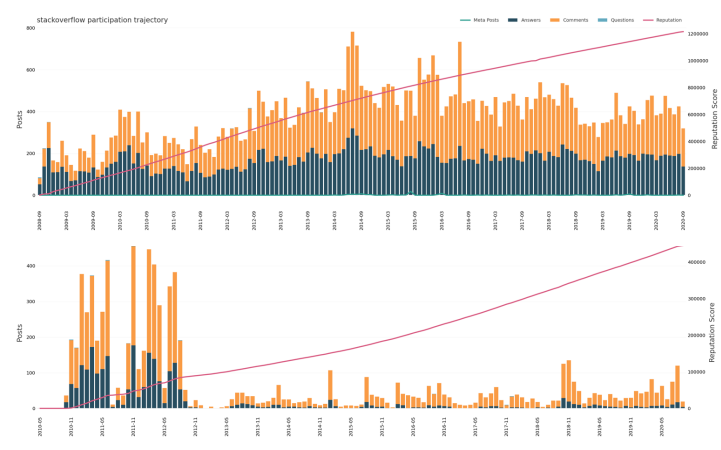

Figure 1. Posts and reputation change over time

Figure 1 shows the contribution of posts and reputation score growth for two of the interviewed users in this study. It is clear from the bars that the user represented in the upper graph has consistently contributed posts throughout their membership on the platform. The darker parts of the bars represent answers per month and the lighter parts represent comments. As is the case with most users, however, this user has only very infrequently contributed questions (shown as a third shade at the top the bars). The user represented in the lower graph shows a similar pattern of contributing mostly answers and comments, but by contrast, this user contributed many posts at the beginning of their membership and then dropped in quantity significantly for the remainder of their time. The continuous 
lines on each graph that indicate reputation score, however, have a similar shape with the line on the lower graph continuing to grow despite the lack of posting activity. This can be described as passive reputation growth, where posts continue to gain score through upvotes long after a user contributes them.

While the mechanics of the platform reward a quick pace of interaction, posts may have very long lives as part of the repository of documents, continuing to reward contributors for years after they are posted in some cases. Over these long lifespans posts are sometimes edited by the original contributor and often by other users. However, given the restrictive caps on reputation score production from edits, other users tend to go unrewarded for their work, though they may receive badges on their profile for editing. This means that in terms of reputation score, it is the original contributor that reaps the benefit of the community maintaining their contribution despite, in some cases, the content of the contribution being completely changed through subsequent updates over a period of years.

\section{CONCLUSION}

In this exploratory paper, our preliminary analysis examines two interrelated particularities of a tension between interacting and documenting as knowledge sharing tasks on Stack Overflow. On the one hand, platform mechanics reward a fast pace of interaction with users quickly answering each other's questions. On the other hand, features such as distributed editing privileges make a slower temporality of knowledge sharing possible where users refine and update information over periods that can span years.

While this tension is particularly visible in the case of Stack Overflow, it is also worth considering that it may be integral to many digital platforms associated with informal learning at scale. Digital platforms rely on the collection of traces of user's activity and in the case of commercial platforms like Stack Overflow, increasing the volume of these traces is often integral to a business model that relies on views and interactions with directed advertising [8]. To increase revenue, commercial knowledge sharing platforms are reliant on a quick pace of interaction with increasing levels of traffic and constant contribution of new posts. On the other hand, to be successful, such platforms also need to support vibrant learning communities where users can not only receive timely answers to their questions, but can also rely on the quality and currency of the knowledge shared. In this sense, we argue that a tension between the knowledge sharing tasks of interacting and documenting is a core concern for understanding platforms as learning contexts. However, while the tension is analytically relevant to the study of informal learning at scale, and a member's concern for users that can be framed in terms of a problem to be overcome, it may be a necessary aspect of the functioning of many knowledge sharing platforms.

\section{ACKNOWLEDGEMENTS}

This research is supported by Swedish Research Council grant 2018-03682.

\section{REFERENCES}

[1] Garfinkel, H. 1967. Studies in Ethnomethodology. Polity.

[2] Geiger, R.S. and Ribes, D. 2011. Trace Ethnography: Following Coordination through Documentary Practices. 2011 44th Hawaii International Conference on System Sciences (Kauai, HI, Jan. 2011), 1-10.

[3] Hudgins, W. et al. 2020. Informal Learning Communities: The Other Massive Open Online "C." Proceedings of the Seventh ACM Conference on Learning@ Scale (Virtual Event USA, Aug. 2020), 91-101.

[4] Kumar, V. and Pedanekar, N.E. 2016. Mining Shapes of Expertise in Online Social Q\&A Communities. Proceedings of the 19th ACM Conference on Computer Supported Cooperative Work and Social Computing Companion - CSCW'16 Companion (San Francisco, California, USA, 2016), 317-320.

[5] Mamykina, L. et al. 2011. Design lessons from the fastest q\&a site in the west. Proceedings of the 2011 annual conference on Human factors in computing systems - CHI '11 (Vancouver, BC, Canada, 2011), 2857.

[6] Mugar, G. et al. 2014. Planet hunters and seafloor explorers: legitimate peripheral participation through practice proxies in online citizen science. Proceedings of the 17th ACM conference on Computer supported cooperative work \& social computing (Baltimore Maryland USA, Feb. 2014), 109-119.

[7] Pai, A.H. et al. 2014. Modeling programming knowledge for mentoring at scale. Proceedings of the first ACM conference on Learning@scale conference (Atlanta Georgia USA, Mar. 2014), 181-182.

[8] Srnicek, N. 2016. Platform Capitalism. Polity.

[9] What is reputation? How do I earn (and lose) it? https://stackoverflow.com/help/whats-reputation. Accessed: 2021-03-30.

[10] Zagalsky, A. et al. 2016. How the R community creates and curates knowledge: a comparative study of stack overflow and mailing lists. Proceedings of the 13th International Workshop on Mining Software Repositories - MSR '16 (Austin, Texas, 2016), 441451. 